nephron

Practice
Nephron 2017;137:256-259

DOI: $10.1159 / 000476078$
Received: March 29, 2017

Accepted after revision: April 20, 2017

Published online: June 9, 2017

\title{
Epigenetic Changes in the Acute Kidney Injury-to-Chronic Kidney Disease Transition
}

\author{
Masaomi Nangaku Yosuke Hirakawa Imari Mimura Reiko Inagi Tetsuhiro Tanaka \\ Division of Nephrology and Endocrinology, The University of Tokyo Graduate School of Medicine, Tokyo, Japan
}

\author{
Keywords \\ Epigenetics · Hypoxia-inducible factor · Hypoxia - Acute \\ kidney injury $\cdot$ Chronic kidney disease $\cdot$ Histone
}

\begin{abstract}
Previously acute kidney injury (AKI) had been believed to be a transient event, and recovery from AKI had been thought to lead to no consequences. However, recent epidemiological studies have shown that even if there is complete recovery of the kidney function, AKI can eventually result in chronic kidney disease (CKD) and eventually in end-stage kidney disease in the long term. Transition of AKI to CKD is mediated by multiple mechanisms, including aberrant cell cycle arrest and hypoxia. Hypoxia of the kidney is induced by rarefaction of the peritubular capillaries after AKI episodes, and induces inflammation and fibrosis. It should also be noted that epigenetic changes are closely related to hypoxia, and epigenetic changes induced by hypoxia, called "hypoxic memory" can explain the AKI-to-CKD transition in the long term after complete recovery from the initial AKI episode. Targeting hypoxia and subsequent epigenetic changes are promising strategies to block the transition from AKI to CKD.
\end{abstract}

(C) 2017 S. Karger AG, Basel

\section{Introduction}

Previously acute kidney injury (AKI) had been believed to be a transient event, and patients who survive an episode of AKI had been thought to regain complete kid- ney function with excellent long-term prognoses. However, recent epidemiological studies and meta-analyses have shown that even if the kidney function is completely restored, AKI can lead to chronic kidney disease (CKD) and eventually result in end-stage kidney disease in the long term. The AKI-to-CKD transition was also supported by a number of animal studies, which showed development of decreased function and fibrosis of the kidney due to maladaptive repair after recovery from AKI [1].

\section{Mechanisms of the AKI-to-CKD Transition}

Mechanisms underlying the AKI-to-CKD transition are a focus of intensive researches. Several mechanisms of the AKI-to-CKD transition have been proposed, such as aberrant cell cycle arrest and hypoxia [2]. Hypoxia is important in the pathogenesis of kidney disease because kidney is physiologically hypoxic primarily due to an arterial-venous diffusional oxygen shunt that permits the kidney to extract no more than $10 \%$ of the oxygen delivered by the renal artery $[3,4]$. Hypoxia of the kidney is induced by rarefaction of the peritubular capillaries, and some capillaries lack perfusion and are nonfunctional after AKI

Contribution from the AKI \& CRRT 2017 Symposium at the 22nd International Conference on Advances in Critical Care Nephrology, Manchester Grand Hyatt, San Diego, CA, USA, March 7-10, 2017. This symposium was supported in part by the NIDDK funded University of Alabama at Birmingham-University of California San Diego O'Brien Center for Acute Kidney Injury Research (P30 DK079337).

\section{KARGER}

(c) 2017 S. Karger AG, Basel 


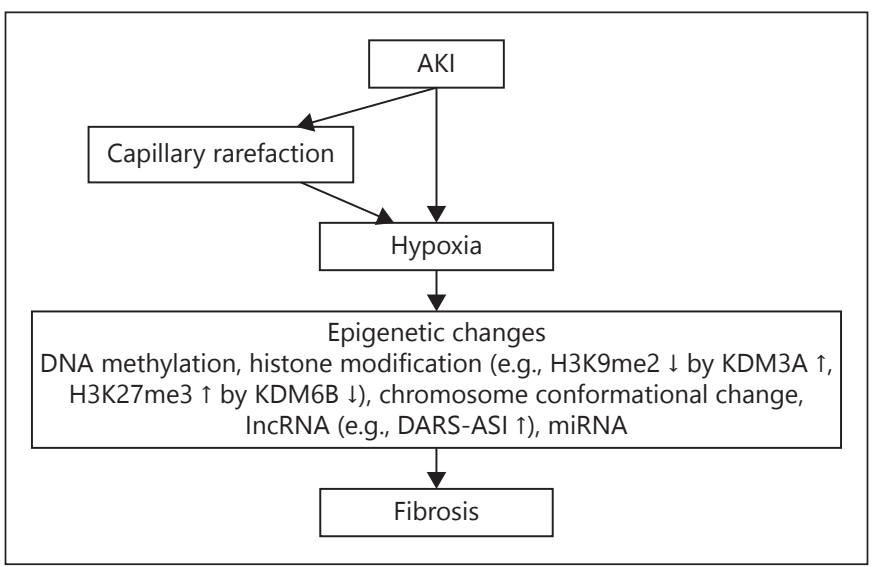

Fig. 1. Schematic view of the AKI-to-CKD transition mediated by hypoxic memory. Hypoxia is recorded as epigenetic changes in the cell and has a long-term effect.

episodes [5]. Hypoxia induces sterile inflammation and fibrosis, and fibrosis, in turn, aggravates hypoxia because fibrosis leads to loss of capillaries and increases the distance between capillaries and resident tubular cells, leading to reduced oxygen diffusion efficiency.

Hypoxia-inducible factor (HIF) serves as a master regulator of adaptive responses against hypoxia. HIF is a dimeric protein complex consisting of alpha (HIF- $\alpha)$ and beta (HIF- $\beta$, also referred to as aryl hydrocarbon receptor nuclear translocator) subunits, and enhances the transcription of $>100$ target genes controlling hematopoiesis (e.g., erythropoietin), angiogenesis (e.g., vascular endothelial growth factor [VEGF]), and anaerobic metabolism (e.g., glucose transporters and glycolytic enzymes).

Levels of HIF are regulated by the oxygen-sensing HIF prolyl hydroxylases (PHD), which belong to the 2-oxoglutarate dependent oxygenase family. Under normoxic conditions, two conserved proline residues in HIF- $\alpha$ are hydroxylated by PHD. Hydroxylated HIF- $\alpha$ is recognized by von Hippel-Lindau tumor suppressor protein, resulting in immediate proteasomal degradation. In contrast, under hypoxic conditions, PHD cannot hydroxylate HIF- $\alpha$, thus leaving HIF- $\alpha$ intact. HIF- $\alpha$ is then able to translocate to the nucleus where it binds and forms a heterodimer with HIF- $\beta$, which is constitutively expressed irrespective of oxygen tension.

To add more clarity to the contribution of inflammation to kidney injury, we studied the role of vascular adhesion protein-1 (VAP-1) in an animal model of AKI. VAP-1 acts as an adhesion molecule as well as an ectoenzyme that catalyzes oxidative deamination of primary amines, generating hydrogen peroxide $\left(\mathrm{H}_{2} \mathrm{O}_{2}\right)$ in the ex-

Epigenetic Changes in the AKI-to-CKD

Transition tracellular space. VAP-1 is expressed predominantly in pericytes, which release enzymatically active VAP-1. Inhibition of VAP-1 decreased neutrophil infiltration and ameliorated renal ischemia-reperfusion injury in rats [6]. We also found that CCAAT/enhancer-binding protein $\delta$ (CEBPD), a transcription factor and inflammatory response gene, is a novel regulator of HIF-1, a master regulator of defensive mechanisms against hypoxia. Mechanistically, CEBPD directly binds to the HIF-1 $\alpha$ promoter and enhances its transcription. Notably, CEBPD is rapidly inducible by inflammatory cytokines, such as IL- $1 \beta$ in an NF- $\kappa B$ dependent manner, which not only increases HIF-1a expression during hypoxia, but is also indispensable for the non-hypoxic induction of HIF-1a [7].

\section{Hypoxic Memory}

Technological advances using high-throughput sequencing have allowed us to determine the expression profile of genes, the binding nature of transcription factors, and histone modifications in a genome-wide manner, and demonstrated that gene expression is intricately regulated by DNA methylation, histone modification, changes in chromosome conformation, long non-coding RNAs, and microRNAs [8]. These epigenetic changes induced by hypoxia are stored in cells as "hypoxic memory" [9], and can induce the AKI-to-CKD transition in the long term after complete recovery from the initial AKI episode (Fig. 1). Studies utilizing animal models of AKI showed that hypoxia-induced epigenetic changes promote proinflammatory and profibrotic gene expression, such as monocyte chemoattractant protein-1, TGF- $\beta 1$, and collagen. In the same context, diabetic milieu induces long-term consequences potentially via epigenetic changes as "metabolic memory" [10].

JmjC-domain-containing histone lysine demethylases (JmjC-KDMs) also belong to the 2-oxoglutarate oxygenase family like $\mathrm{PHD}$, and they play a key role in epigenetic changes via modulating the methylation levels of histone tails. We previously performed genome-wide analysis of HIF1 binding sites (chromatin immunoprecipitation with deep sequencing) of endothelial cells exposed to hypoxia and observed that lysine $(\mathrm{K})$-specific demethylase $3 \mathrm{~A}$ is recruited to the GLUT3 (SLC2A3) locus in an HIF1dependent manner, demethylates $\mathrm{H} 3 \mathrm{~K} 9 \mathrm{me} 2$, and induces chromosomal conformational changes so as to upregulate its expression [11].

We also exposed tubular cells to hypoxia and identified novel HIF-1 downstream epigenetic factors that may 
play important roles in the kidney. Our RNA-seq identified lncRNAs that are upregulated under hypoxic condition, and chromatin immunoprecipitation-seq analysis demonstrated that HIF-1 also binds to the lncRNAs under hypoxia. We identified a novel lncRNA, DARS-AS1 (aspartyl-tRNA synthetase anti-sense 1), which is upregulated only under hypoxia in an HIF-1-dependent manner and inhibits apoptotic cell death in renal tubular cells [12].

\section{Treatment Targeting the AKI-to-CKD Transition}

Treatments that successfully decrease the severity of AKI should block the transition from AKI to CKD because AKI severity is associated with subsequent development of CKD. Studies utilizing HIF knockdown mice showed aggravation of AKI, suggesting that HIF activation can serve as a therapeutic target for AKI [13]. To support this, various pharmacological approaches to PHD inhibition improved AKI models, at least as preventive strategies. Animal studies showed that HIF activation prevented the AKI-to-CKD transition. PHD inhibition not only reduced the severity of I/R-induced AKI, which should effectively suppress the AKI-to-CKD transition, but also blocked the mechanisms of the AKI-to-CKD transition itself, probably by promoting repair of renal I/R injury. Various PHD inhibitors are now in clinical trials of anemia in CKD and are expected to be available at the bedside soon [14]. It remains unknown about which HIF target genes are responsible for providing protection in AKI; however, and we need more in-depth knowledge to apply HIF augmentation strategy in AKI patients.

Treatment to suppress the AKI-to-CKD transition can target the amelioration of capillary rarefaction. Treatment with VEGF-121 attenuated the loss of peritubular capillaries with subsequent suppression of the AKI-toCKD transition and fibrotic changes in a rat $I / R$ injury model, although VEGF-121 did not affect acute kidney damage. Another angiogenic factor, angiopoietin-1, also preserved peritubular capillaries and blocked the AKI-toCKD transition. Thus, the preservation of peritubular capillaries early in the AKI process leads to the maintenance of oxygen levels in the kidney, protecting the kidney from the AKI-to-CKD transition.

Another fascinating approach against the AKI-toCKD transition is to target epigenetic changes directly [15]. Recent studies targeting a histone methyltransferase that induces histone $\mathrm{H} 3$ lysine 27 trimethylation showed that pharmacologic inhibition of the histone methyl-

transferase improved kidney fibrosis in a model of unilateral ureteral obstruction [16]. We also found that pharmacologic intervention targeting histone modification can improve kidney fibrosis in a model of the AKI-toCKD transition (Mimura, Hirakawa, Tanaka, Nangaku: manuscript in preparation). However, specificity of genomic sites targeted by epigenetic intervention can be an issue, and epigenetic therapies in kidney disease need more investigations in the future.

\section{Summary}

Hypoxia serves as a key player in AKI pathophysiology and is the final common pathway from CKD to ESKD. Hypoxia also plays an important role in the AKI-to-CKD transition. Epigenetic changes induced by hypoxia in the kidney are likely to be crucial in the pathophysiology of the AKI-to-CKD transition as "hypoxic memory." Therapeutic strategies targeting hypoxia, such as HIF activation, and those targeting epigenetic changes will be effective in blocking the AKI-to-CKD transition.

\section{Acknowledgments}

This work was supported by a Grant-in-Aid for Scientific Research on Innovative Areas 26111003 (M.N.), and Grants-in-Aid for Scientific Research (B) 15H04835 (M.N.) by Japan Society for the Promotion of Science.

\section{Disclosure Statement}

The authors have no conflicts of interest to declare.

References

Nephron 2017;137:256-259 DOI: $10.1159 / 000476078$
1 Basile DP, Bonventre JV, Mehta R, Nangaku M, Unwin R, Rosner MH, Kellum JA, Ronco C; ADQI XIII Work Group: Progression after AKI: understanding maladaptive repair processes to predict and identify therapeutic treatments. J Am Soc Nephrol 2016;27:687-697.

2 Tanaka S, Tanaka T, Nangaku M: Hypoxia as a key player in the AKI-to-CKD transition. Am J Physiol Renal Physiol 2014;307:F1187F1195.

3 Mimura I, Nangaku M: The suffocating kidney: tubulointerstitial hypoxia in end-stage renal disease. Nat Rev Nephrol 2010;6:667678.

4 Nangaku M: Chronic hypoxia and tubulointerstitial injury: a final common pathway to end-stage renal failure. J Am Soc Nephrol 2006; $17: 17-25$. 
5 Hirakawa Y, Yoshihara T, Kamiya M, Mimura I, Fujikura D, Masuda T, Kikuchi R, Takahashi I, Urano Y, Tobita S, Nangaku M: Quantitating intracellular oxygen tension in vivo by phosphorescence lifetime measurement. Sci Rep 2015;5:17838.

6 Tanaka S, Tanaka T, Kawakami T, Takano $\mathrm{H}$, Sugahara M, Saito H, Higashijima Y, Yamaguchi J, Inagi R, Nangaku M: Vascular adhesion protein-1 enhances neutrophil infiltration by generation of hydrogen peroxide in renal ischemia/reperfusion injury. Kidney Int 2017;pii:S0085-2538(17)30041-8.

7 Yamaguchi J, Tanaka T, Eto N, Nangaku M: Inflammation and hypoxia linked to renal injury by CCAAT/enhancer-binding protein $\delta$. Kidney Int 2015;88:262-275.

8 Mimura I, Kanki Y, Kodama T, Nangaku M: Revolution of nephrology research by deep sequencing: ChIP-seq and RNA-seq. Kidney Int 2014;85:31-38.

9 Mimura I, Tanaka T, Nangaku M: New insights into molecular mechanisms of epigenetic regulation in kidney disease. Clin Exp Pharmacol Physiol 2016;43:1159-1167.
10 Hirakawa Y, Tanaka T, Nangaku M: Mechanisms of metabolic memory and renal hypoxia as a therapeutic target in diabetic kidney disease. J Diabetes Investig 2017;8:261-271.

11 Mimura I, Nangaku M, Kanki Y, Tsutsumi S, Inoue T, Kohro T, Yamamoto S, Fujita T, Shimamura T, Suehiro J, Taguchi A, Kobayashi $\mathrm{M}$, Tanimura $\mathrm{K}$, Inagaki $\mathrm{T}$, Tanaka $\mathrm{T}$, Hamakubo T, Sakai J, Aburatani H, Kodama T, Wada Y: Dynamic change of chromatin conformation in response to hypoxia enhances the expression of GLUT3 (SLC2A3) by cooperative interaction of hypoxia-inducible factor 1 and KDM3A. Mol Cell Biol 2012;32: 3018-3032.

12 Mimura I, Hirakawa Y, Kanki Y, Kushida N, Nakaki R, Suzuki Y, Tanaka T, Aburatani H, Nangaku M: Novel lnc RNA regulated by HIF-1 inhibits apoptotic cell death in the renal tubular epithelial cells under hypoxia. Physiol Rep 2017;5:pii:e13203.
13 Kojima I, Tanaka $\mathrm{T}$, Inagi $\mathrm{R}$, Kato $\mathrm{H}$, Yamashita T, Sakiyama A, Ohneda O, Takeda N, Sata M, Miyata T, Fujita T, Nangaku M: Protective role of hypoxia-inducible factor-2alpha against ischemic damage and oxidative stress in the kidney. J Am Soc Nephrol 2007; 18:1218-1226.

14 Sugahara M, Tanaka T, Nangaku M: Prolyl hydroxylase domain inhibitors as a novel therapeutic approach against anemia in chronic kidney disease. Kidney Int, in press.

15 Mimura I, Tanaka T, Nangaku M: Novel therapeutic strategy with hypoxia-inducible factors via reversible epigenetic regulation mechanisms in progressive tubulointerstitial fibrosis. Semin Nephrol 2013;33:375382 .

16 Zhou X, Zang X, Ponnusamy M, Masucci MV, Tolbert E, Gong R, Zhao TC, Liu N, Bayliss G, Dworkin LD, Zhuang S: Enhancer of zeste homolog 2 inhibition attenuates renal fibrosis by maintaining Smad7 and phosphatase and tensin homolog expression. J Am Soc Nephrol 2016;27:2092-2108. 